\title{
Asymptotic Stability of Solutions of Lotka-Volterra Predator-Prey Model for Four Species
}

\author{
A. A. Soliman ${ }^{*}$, E. S. Al-Jarallah² \\ ${ }^{1}$ Department of Mathematics, Faculty of Sciences, Benha University, Benha, Egypt \\ ${ }^{2}$ Department of Mathematics, Faculty of Education, Al Jouf University, Al-Jawf, Kingdom of Saudi Arabia \\ Email: ${ }^{\text {a }}$ a soliman@hotmail.com
}

Received 1 March 2015; accepted 21 April 2015; published 22 April 2015

Copyright (C) 2015 by authors and Scientific Research Publishing Inc.

This work is licensed under the Creative Commons Attribution International License (CC BY). http://creativecommons.org/licenses/by/4.0/

c) (i) Open Access

\begin{abstract}
In this paper, we consider Lotka-Volterra predator-prey model between one and three species. Two cases are distinguished. The first is Lotka-Volterra model of one prey-three predators and the second is Lotka-Volterra model of one predator-three preys. The existence conditions of nonnegative equilibrium points are established. The local stability analysis of the system is carried out.
\end{abstract}

\section{Keywords}

Lotka-Volterra, Prey-Predators, Species, Equilibrium Points Stability, Locally Asymptotically Stable, Globally Asymptotically Stable, Unstable

\section{Introduction}

The Lotka-Volterra model provides a nice mathematical device to study and understand complex systems of mutually interacting species or agent [1]. In the past decades, Lotka-Volterra type systems have been extensively investigated, especially in biology and ecology [2]-[8]. A basic issue addressed in the studies concerns stability property of the systems because of its relevance to the coexistence of different species in a community [9]. It turns out that the stability of a Lotka-Volterra system relies crucially on the interaction matrix of the system.

A Lotka-Volterra system of $n$-dimensions is expressed by the ordinary differential equations [4] [10]:

$$
\dot{x}_{i}(t)=x_{i}(t)\left(b_{i}-\sum_{j=1}^{n} a_{i j} x_{j}(t)\right), \quad i \in N .
$$

"Corresponding author. 
where $N=\{1,2, \cdots, n\}$ and $n$ is the species number. In (1.1), the function $x_{i}(t)$ represents the density of species $i$ at time $t$, the constant $b_{i}$, is the carrying capacity of species $i$, and $a_{i j}$ represents the effect of interspecific (if $i \neq j$ ) or intraspecific (if $i=j$ ) interaction. In vector form, System (1.1) is expressed as

$$
\dot{x}=X(b-A x),
$$

where $x=\operatorname{col}\left(x_{1}, x_{2}, \cdots, x_{n}\right)$ is an $n$-dimensional state vector, $X=\operatorname{diag}\left(x_{1}, x_{2}, \cdots, x_{n}\right)$ is an $n \times n$ diagonal matrix, $b=\operatorname{col}\left(b_{1}, b_{2}, \cdots, b_{n}\right)$ is an $n$-dimensional real vector, and $A=\left(a_{i j}\right)$ is an $n \times n$ community matrix.

The existence and stability of a nonnegative equilibrium point of system (1.1) or subsystems of (1.1) has been investigated by many authors [9] [11] and [12]. The global stability of system (1.1) has been studied by many authors [9] [11] [13]-[16].

In this paper, we shall concentrate on Lotka-Volterra systems of the fourth dimension. A Lotka-Volterra two preys-two predators system is studied by Takeuchi and Adachi [15], and [16]. The first is Lotka-Volterra model of one prey-three predators and the second is Lotka-Volterra model of three prey-one predator.

This work is organized as follows: In Section 2, we describe our model. In Section 3, the existence conditions of nonnegative equilibrium points are established. The local stability analysis of the system is carried out in Section 4.

In Section 5, we present an example to clarify each case.

\section{The Model}

\section{Lotka-Volterra Model}

In this section we consider Lotka-Volterra predator-prey model between one and three species and assume that there is no interspicific competition between the three species $x_{2}, x_{3}$ and $x_{4}$. This is represented by the following system of differential equations:

$$
\left.\begin{array}{l}
x_{1}^{\prime}(t)=x_{1}(t)\left[b_{1}-a_{11} x_{1}(t)-a_{12} x_{2}(t)-a_{13} x_{3}(t)-a_{14} x_{4}(t)\right], \\
x_{2}^{\prime}(t)=x_{2}(t)\left[b_{2}-a_{21} x_{1}(t)-a_{22} x_{2}(t)\right], \\
x_{3}^{\prime}(t)=x_{3}(t)\left[b_{3}-a_{31} x_{1}(t)-a_{33} x_{3}(t)\right], \\
x_{4}^{\prime}(t)=x_{4}(t)\left[b_{4}-a_{41} x_{1}(t)-a_{44} x_{4}(t)\right] .
\end{array}\right\}
$$

where $x_{i}(t),(i=1,2,3,4)$ represents the density of species $i$ at time $t$, the constant $b_{i}$ is the carrying capacity of species $i$ and $a_{i j}$ represents the effect of interspecific (if $i \neq j$ ) or intraspecific (if $i=j$ ) interaction. In vector form, system (2.1) is expressed as:

$$
\dot{x}=X(b-A x),
$$

where $x=\operatorname{col}\left(x_{1}, x_{2}, x_{3}, x_{4}\right)$ is a 4-dimensional state vector, $X=\operatorname{diag}\left(x_{1}, x_{2}, x_{3}, x_{4}\right)$ is a $4 \times 4$ diagonal matrix, $b=\operatorname{col}\left(b_{1}, b_{2}, b_{3}, b_{4}\right)$ is a 4-dimnsional real vector, and

$$
A=\left(\begin{array}{cccc}
a_{11} & a_{12} & a_{13} & a_{14} \\
a_{21} & a_{22} & 0 & 0 \\
a_{31} & 0 & a_{33} & 0 \\
a_{41} & 0 & 0 & a_{44}
\end{array}\right) .
$$

is a $4 \times 4$ community matrix.

The system (2.1) is a prey-predator system if the following assumption is satisfied.

(H1) $a_{i i}>0, a_{1 j} a_{j 1}<0, i=1,2,3,4, j=1,2,3$.

Two cases of system (2.1) can be distinguished:

The first case describes a one prey-three predators system where $x_{1}$ represents the prey and $x_{2}, x_{3}, x_{4}$ represent the predators. In this case we assume that the following conditions are satisfied in addition to (H1):

(H2) $a_{1 j}>0, a_{j 1}<0, j=2,3,4$.

(H3) $b_{1}>0, b_{j}<0, j=2,3,4$. 
The second case describes a one predator-three preys system where $x_{1}$ represents the predator and $x_{2}, x_{3}, x_{4}$ represent the preys. In this case we assume that the following conditions are satisfied in addition to (H1).

(H2)' $a_{1 j}<0, a_{j 1}>0, j=2,3,4$,

(H3)' $b_{1}<0, b_{j}>0, j=2,3,4$.

\section{Equilibrium Analysis}

\subsection{Existence of the Quilibrium Points}

In this section, the existence of the equilibrium points of system (2.1) in each case is investigated. At most there are nine possible non-negative equilibrium points for system (2.1) in the first case, the existence conditions of them are given as the following:

1) The equilibrium points $E_{0}=(0,0,0,0)$ and $E_{1}=\left(\frac{b_{1}}{a_{11}}, 0,0,0\right)$ are always exist where $E_{1}$ is the equilibrium point in the absence of predation and $\frac{b_{1}}{a_{11}}>0$ according to conditions (H1) and (H3).

2) The positive equilibrium point $E_{1 j}=\left(\tilde{x}_{1 j}, \tilde{x}_{j}\right), j=2,3,4$ exists in the first quadrant of the $x_{1} x_{j}$ plane if and only if the following condition is satisfied

(H4): $\frac{b_{1}}{b_{j}}<\frac{a_{11}}{a_{j 1}}, j=2,3,4$,

where $\tilde{x}_{1 j}$ and $\tilde{x}_{j}$ are given by

$$
\left.\begin{array}{l}
\tilde{x}_{1 j}=\frac{a_{j j} b_{1}-a_{1 j} b_{j}}{a_{11} a_{j j}-a_{1 j} a_{j 1}}, \\
\tilde{x}_{j}=\frac{a_{11} b_{j}-a_{j 1} b_{1}}{a_{11} a_{j j}-a_{1 j} a_{j 1}}, j=2,3,4 .
\end{array}\right\}
$$

3) The positive equilibrium point $E_{1 j k}=\left(\hat{x}_{1 j k}, \hat{x}_{j k}, \hat{x}_{k j}\right), j=2,3, k=3,4, j \neq k$ exists in the first octant of $x_{1} x_{j} x_{k}$ space if and only if the following conditions are satisfied:

(H5): $\quad b_{j}>a_{j 1}\left(\frac{a_{k k} b_{1}-a_{1 k} b_{k}}{a_{11} a_{k k}-a_{1 k} a_{k 1}}\right)=a_{j 1} \tilde{x}_{1 k}$,

$$
\begin{aligned}
& b_{k}>a_{k 1}\left(\frac{a_{j j} b_{1}-a_{1 j} b_{j}}{a_{11} a_{j j}-a_{1 j} a_{j 1}}\right)=a_{k 1} \tilde{x}_{1 j}, \\
& j=2,3, k=3,4, j \neq k .
\end{aligned}
$$

where $\hat{x}_{1 j k}, \hat{x}_{j k}$ and $\hat{x}_{k j}$ are given by

$$
\left.\begin{array}{l}
\hat{x}_{1 j k}=\frac{a_{j j} a_{k k} b_{1}-a_{1 j} a_{k k} b_{j}-a_{1 k} a_{j j} b_{k}}{a_{11} a_{j j} a_{k k}-a_{1 j} a_{j 1} a_{k k}-a_{1 k} a_{k 1} a_{j j}}, \\
\hat{x}_{j k}=\frac{-a_{j 1} a_{k k} b_{1}-a_{1 k} a_{k 1} b_{j}+a_{11} a_{k k} b_{j}+a_{1 k} a_{j 1} b_{k}}{a_{11} a_{j j} a_{k k}-a_{1 j} a_{j 1} a_{k k}-a_{1 k} a_{k 1} a_{j j}}, \\
\hat{x}_{k j}=\frac{-a_{j j} a_{k 1} b_{1}+a_{1 j} a_{k 1} b_{j}-a_{1 j} a_{j 1} b_{k}+a_{11} a_{j j} b_{k}}{a_{11} a_{i j} a_{k k}-a_{1 j} a_{j 1} a_{k k}-a_{1 k} a_{k 1} a_{j j}}, \\
j=2,3, \quad k=3,4, \quad j \neq k .
\end{array}\right\}
$$

4) The positive equilibrium point $E^{*}=\left(x_{1}^{*}, x_{2}^{*}, x_{3}^{*}, x_{4}^{*}\right)$ exists in the positive cone (nonnegative octant) $R_{+}^{4}=\left\{x=\left(x_{1}, x_{2}, x_{3}, x_{4}\right) \in R^{4} \mid x_{i} \geq 0, i=1,2,3,4\right\}$. if and only if the following conditions are satisfied 
(H6) $b_{2}>a_{21}\left(\frac{a_{33} a_{44} b_{1}-a_{13} a_{44} b_{3}-a_{14} a_{33} b_{4}}{a_{11} a_{33} a_{44}-a_{13} a_{31} a_{44}-a_{14} a_{41} a_{33}}\right)=a_{21} \hat{x}_{134}$,

$$
\begin{aligned}
& b_{3}>a_{31}\left(\frac{a_{22} a_{44} b_{1}-a_{14} a_{22} b_{4}-a_{12} a_{44} b_{2}}{a_{11} a_{22} a_{44}-a_{12} a_{21} a_{44}-a_{14} a_{41} a_{22}}\right)=a_{31} \hat{x}_{124}, \\
& b_{4}>a_{41}\left(\frac{a_{22} a_{33} b_{1}-a_{12} a_{33} b_{2}-a_{13} a_{22} b_{3}}{a_{11} a_{22} a_{33}-a_{12} a_{21} a_{33}-a_{13} a_{31} a_{22}}\right)=a_{41} \hat{x}_{123} .
\end{aligned}
$$

where $x_{i}^{*}, i=1,2,3,4$ are given by

$$
x_{i}^{*}=\frac{\bar{x}_{i}}{|A|}, i=1,2,3,4
$$

where

$$
\begin{aligned}
& \bar{x}_{1}=a_{22} a_{33} a_{44} b_{1}-a_{12} a_{33} a_{44} b_{2}-a_{13} a_{22} a_{44} b_{3}-a_{14} a_{22} a_{33} b_{4}, \\
& \bar{x}_{2}=-a_{21} a_{33} a_{44} b_{1}+\left(a_{11} a_{33} a_{44}-a_{14} a_{41} a_{33}-a_{13} a_{31} a_{44}\right) b_{2}+a_{13} a_{21} a_{44} b_{3}+a_{14} a_{21} a_{33} b_{4}, \\
& \bar{x}_{3}=-a_{22} a_{31} a_{44} b_{1}+a_{12} a_{31} a_{44} b_{2}+\left(a_{11} a_{22} a_{44}-a_{12} a_{21} a_{44}-a_{14} a_{41} a_{22}\right) b_{3}+a_{14} a_{22} a_{31} b_{4}, \\
& \bar{x}_{4}=-a_{22} a_{33} a_{41} b_{1}+a_{12} a_{33} a_{41} b_{2}+a_{13} a_{22} a_{41} b_{3}+\left(a_{11} a_{22} a_{33}-a_{12} a_{21} a_{33}-a_{13} a_{31} a_{22}\right) b_{4}, \\
& |A|=a_{11} a_{22} a_{33} a_{44}-a_{12} a_{21} a_{33} a_{44}-a_{13} a_{31} a_{22} a_{44}-a_{14} a_{41} a_{22} a_{33} .
\end{aligned}
$$

$A$ is the interaction matrix defined in (2.2).

For the second case of system (2.1), at most there are fifteen possible nonnegative equilibrium points. The existence conditions of them are given as the following:

1) The equilibrium points

$$
E_{0}=(0,0,0,0), E_{2}=\left(0, \frac{b_{2}}{a_{22}}, 0,0\right), E_{3}=\left(0,0, \frac{b_{3}}{a_{33}}, 0\right) \text { and } E_{4}=\left(0,0,0, \frac{b_{4}}{a_{44}}\right)
$$

are always exist where $E_{2}, E_{3}, E_{4}$ are the equilibrium points in the absence of predation and $\frac{b_{j}}{a_{j j}}>0,(j=2,3,4)$ according to conditions (H1) and (H3)'.

2) The positive equilibrium point $E_{1 j}=\left(\tilde{x}_{1 j}, \tilde{x}_{j}\right), j=2,3,4$ exists in the first quadrant of $x_{1} x_{j}$ plane if and only if the following condition is satisfied

(H4)': $\frac{b_{1}}{b_{j}}>\frac{a_{1 j}}{a_{j j}}, j=2,3,4$.

where $\hat{x}_{1 j}$ and $\hat{x}_{j}$ are given by (3.1).

3) In the absence of predator and one prey species, both the other two prey species grow. Thus, the equilibrium point $E_{j k}=\left(\frac{b_{j}}{a_{j j}}, \frac{b_{k}}{a_{k k}}\right), j=2,3, k=3,4, j \neq k$ always exists in the interior of $x_{j} x_{k}$ plane where $\frac{b_{j}}{a_{j j}}>0$, $\frac{b_{k}}{a_{k k}}>0$ according to conditions (H1) and (H3)'.

4) The positive equilibrium point $E_{1 j k}=\left(\hat{x}_{1 j k}, \hat{x}_{j k}, \hat{x}_{k j}\right), j=3,4, k=3,4, j \neq k$ exists in the first octant of $x_{1} x_{j} x_{k}$ space if and only if the following conditions are satisfied

(H5)' $\quad b_{1}>\frac{a_{1 j}}{a_{j j}} b_{j}+\frac{a_{1 k}}{a_{k k}} b_{k}$,

$$
\begin{aligned}
& b_{j}>a_{j 1}\left(\frac{a_{k k} b_{1}-a_{1 k} b_{k}}{a_{11} a_{k k}-a_{1 k} a_{k 1}}\right)=a_{j 1} \tilde{x}_{1 k}, \\
& b_{k}>a_{k 1}\left(\frac{a_{j j} b_{1}-a_{1 j} b_{j}}{a_{11} a_{j j}-a_{1 j} a_{j 1}}\right)=a_{k 1} \tilde{x}_{1 j} .
\end{aligned}
$$


where $\hat{x}_{1 j k}, \hat{x}_{j k}$ and $\hat{x}_{k j}$ are given by (3.2).

5) In the absence of predator, all three prey species grow. Thus, the positive equilibrium point $E_{234}=\left(\frac{b_{2}}{a_{22}}, \frac{b_{3}}{a_{33}}, \frac{b_{4}}{a_{44}}\right)$ always exists in the interior of $x_{2} x_{3} x_{4}$ space.

6) The positive equilibrium point $E^{*}=\left(x_{1}^{*}, x_{2}^{*}, x_{3}^{*}, x_{4}^{*}\right)$ exists in $R_{+}^{4}$ if and only if the following conditions are satisfied

$$
\begin{aligned}
\text { (H6)': } b_{1} & >\frac{a_{12}}{a_{22}} b_{2}+\frac{a_{13}}{a_{33}} b_{3}+\frac{a_{14}}{a_{44}} b_{4}, \\
b_{2} & >a_{21}\left(\frac{a_{33} a_{44} b_{1}-a_{13} a_{44} b_{3}-a_{14} a_{33} b_{4}}{a_{11} a_{33} a_{44}-a_{13} a_{31} a_{44}-a_{14} a_{41} a_{33}}\right)=a_{21} \hat{x}_{134}, \\
b_{3} & >a_{31}\left(\frac{a_{22} a_{44} b_{1}-a_{14} a_{22} b_{4}-a_{12} a_{44} b_{2}}{a_{11} a_{22} a_{44}-a_{12} a_{21} a_{44}-a_{14} a_{41} a_{22}}\right)=a_{31} \hat{x}_{124}, \\
b_{4} & >a_{41}\left(\frac{a_{22} a_{33} b_{1}-a_{12} a_{33} b_{2}-a_{13} a_{22} b_{3}}{a_{11} a_{22} a_{33}-a_{12} a_{21} a_{33}-a_{13} a_{31} a_{22}}\right)=a_{41} \hat{x}_{123} .
\end{aligned}
$$

where $x_{i}^{*}, i=1,2,3,4$ are given by (3.3).

\subsection{Remark}

We will use the symbols $E_{1200}, E_{1030}$ and $E_{1004}$ to denote the nonnegative equilibrium points $\left(\tilde{x}_{12}, \tilde{x}_{2}, 0,0\right)$, $\left(\tilde{x}_{13}, 0, \tilde{x}_{3}, 0\right)$ and $\left(\tilde{x}_{14}, 0,0, \tilde{x}_{4}\right)$ respectively, where $\tilde{x}_{1 j}$ and $\tilde{x}_{j}, j=2,3,4$ are given by (3.1), the symbols $E_{1230}, E_{1204}$ and $E_{1034}$ to denote the nonnegative equilibrium points $\left(\hat{x}_{123}, \hat{x}_{23}, \hat{x}_{32}, 0\right),\left(\hat{x}_{124}, \hat{x}_{24}, 0, \hat{x}_{42}\right)$ and $\left(\hat{x}_{134}, 0, \hat{x}_{34}, \hat{x}_{43}\right)$ respectively, where $\hat{x}_{1 j k}, \hat{x}_{j k}, \hat{x}_{k j}, j=1,2, k=3,4, j \neq k$ are given by (3.2) and use the symbols $E_{0230}, E_{0204}$ and $E_{0034}$ to denote the nonnegative equilibrium points $\left(0, \frac{b_{2}}{a_{22}}, \frac{b_{3}}{a_{33}}, 0\right),\left(0, \frac{b_{2}}{a_{22}}, 0, \frac{b_{4}}{a_{44}}\right)$ and $\left(0,0, \frac{b_{3}}{a_{33}}, \frac{b_{4}}{a_{44}}\right)$ respectively.

\section{Stability Analysis}

\subsection{Stability of Equilibrium Points}

In this section, the local stability analysis of equilibrium points is investigated. Assuming that all previous equilibrium points existing.

The Jacobian matrix $J$ of system (2.1) is given by:

$$
J=\left[\begin{array}{cccc}
b_{1}-2 a_{11} x_{1}-a_{12} x_{2} & -a_{12} x_{1} & -a_{13} x_{1} & -a_{14} x_{1} \\
-a_{13} x_{3}-a_{14} x_{4} & b_{2}-a_{21} x_{1} & 0 & 0 \\
-a_{21} x_{2} & -2 a_{22} x_{2} & 0 & \\
& 0 & b_{3}-a_{31} x_{1} & 0 \\
-a_{31} x_{3} & & -2 a_{33} x_{3} & \\
& 0 & 0 & b_{4}-a_{41} x_{1} \\
-a_{41} x_{4} & & & -2 a_{44} x_{4}
\end{array}\right] .
$$

Computing the variation matrixes corresponding to each equilibrium point and then using Routh-Hurwitz criteria [17], the following results can be observed:

1) Substituting by $E_{0}$ in the variation matrix (4.1), we get the eigenvalues $\lambda_{i}=b_{i}, i=1,2,3,4$.

So for the first case, $E_{0}$ is a saddle point with locally stable manifold in the $x_{2} X_{3} X_{4}$ space and with unstable 
manifold in the $x_{1}$ direction. Near $E_{0}$ the prey's population $x_{1}$ grows while the predators' populations $x_{2}, x_{3}$ and $x_{4}$ decline.

For the second case, $E_{0}$ is a saddle point with locally stable manifold in the $x_{1}$ direction and with unstable manifold in the $x_{2} x_{3} x_{4}$ space. Near $E_{0}$ the predator population $x_{1}$ decline while the preys' populations $x_{2}, x_{3}$ and $x_{4}$ grow.

2) Substituting by $E_{1}$ in the variation matrix (4.1), we get the eigenvalues $\lambda_{1}=-b_{1}<0, \quad \lambda_{j}=b_{j}-\frac{a_{1 j}}{a_{11}} b_{1}>0$, $j=2,3,4$.

(By using (H3) and (H4)).

So $E_{1}$ is a saddle point with locally stable manifold in the $x_{1}$ direction and with unstable manifold in the $x_{2} X_{3} X_{4}$ space. Near $E_{1}$ the prey species $x_{1}$ remains close to $\frac{b_{1}}{a_{11}}$.

Similarly, $E_{2}$ has three positive eigenvalues

$$
\lambda_{1}=b_{1}-\frac{a_{12}}{a_{22}} b_{2}>0, \quad \lambda_{2}=-b_{2}<0, \quad \lambda_{3}=b_{3}>0, \quad \lambda_{3}=b_{3}>0 .
$$

(By using (H3)' and (H4)')

So $E_{2}$ is a saddle point with locally stable manifold in the $x_{2}$ direction and with unstable manifold in the $x_{1} x_{3} X_{4}$ space. Near $E_{2}$ the prey species $x_{2}$ remains close to $\frac{b_{2}}{a_{22}}$.

$E_{3}$ and $E_{4}$ have the same stability behavior of $E_{2}$.

We now state the local stability behavior of other equilibrium points in the form of Theorems. The proofs of these theorems follow directly from the Routh-Hurwitz criteria [12].

\section{Theorem 4.1}

1) $E_{12}$ is locally asymptotically stable in the $x_{1} x_{2}$ plane.

2) If $E_{1230}$ and $E_{1204}$ exist, then $E_{1200}$ is a saddle point with locally stable manifold in the $x_{1} x_{2}$ plane and with unstable manifold in the $x_{3} x_{4}$ plane.

\section{Proof}

Consider the following subsystem from (2.1)

$$
\left.\begin{array}{l}
x_{1}^{\prime}(t)=x_{1}(t)\left[b_{1}-a_{11} x_{1}(t)-a_{12} x_{2}(t)\right], \\
x_{2}^{\prime}(t)=x_{2}(t)\left[b_{2}-a_{21} x_{1}(t)-a_{22} x_{2}(t)\right] .
\end{array}\right\}
$$

Evaluating the variation matrix of system (4.2) at $E_{12}$, we have

The characteristic polynomial is

$$
M_{12}=\left(\begin{array}{ll}
-a_{11} \tilde{x}_{12} & -a_{12} \tilde{x}_{12} \\
-a_{21} \tilde{x}_{12} & -a_{22} \tilde{x}_{2}
\end{array}\right) .
$$

$$
\lambda^{2}+\left(a_{11} \tilde{X}_{12}+a_{22} \tilde{X}_{2}\right) \lambda+\left(a_{11} a_{22}-a_{12} a_{21}\right) \tilde{X}_{12} \tilde{X}_{2} .
$$

Since

$$
\begin{aligned}
& \operatorname{trace}\left(M_{12}\right)=\lambda_{1}+\lambda_{2}=-\left(a_{11} \tilde{X}_{12}+a_{22} \tilde{X}_{2}\right)<0, \\
& \operatorname{det}\left(M_{12}\right)=\lambda_{1} \lambda_{2}=\left(a_{11} a_{22}-a_{12} a_{21}\right) \tilde{x}_{12} \tilde{X}_{2}>0 .
\end{aligned}
$$

Then, $\lambda_{1}$ and $\lambda_{2}$ have negative real parts. Thus, $E_{12}$ is locally asymptotically stable in the $x_{1} x_{2}$ plane. Computing the variation matrix (4.1) at $E_{1200}$, we have

$$
J_{12}=\left[\begin{array}{cccc}
-a_{11} \tilde{x}_{12} & -a_{12} \tilde{x}_{12} & -a_{13} \tilde{x}_{12} & -a_{14} \tilde{x}_{12} \\
-a_{21} \tilde{x}_{2} & -a_{22} \tilde{x}_{2} & 0 & 0 \\
0 & 0 & b_{3}-a_{31} \tilde{x}_{12} & 0 \\
0 & 0 & 0 & b_{4}-a_{41} \tilde{x}_{12}
\end{array}\right]
$$


The characteristic equation of matrix $V_{12}$ is

$$
\left[\lambda^{2}+\left(a_{11} \tilde{x}_{12}+a_{22} \tilde{x}_{2}\right) \lambda+\left(a_{11} a_{22}-a_{12} a_{21}\right) \tilde{x}_{12} \tilde{x}_{2}\right]\left(b_{3}-a_{31} \tilde{x}_{12}-\lambda\right)\left(b_{4}-a_{41} \tilde{x}_{12}-\lambda\right)=0 .
$$

Comparing with (4.3) we get that $\lambda_{1}$ and $\lambda_{2}$ have negative real parts and

$$
\lambda_{3}=b_{3}-a_{31} \tilde{x}_{12}, \quad \lambda_{4}=b_{4}-a_{41} \tilde{x}_{12} .
$$

If $E_{1230}$ and $E_{1204}$ exist, then $\lambda_{3}>0$ and $\lambda_{4}>0$ (by using (H5) and (H5)').

Therefore, $E_{1200}$ is a saddle point with locally stable manifold in the $x_{1} x_{2}$ plane and with unstable manifold in the $x_{3} x_{4}$ plane.

\subsection{Remark}

1) Behavior of solutions near the equilibrium points $E_{13}$ and $E_{14}$ are the same behavior of solutions near the equilibrium point $E_{12}$.

2) Behavior of solutions near $E_{1030}$ and $E_{1004}$ are the same behavior of solutions near $E_{1200}$.

\section{Theorem 4.1}

a) $E_{23}$ is locally asymptotically stable in the $x_{2} x_{3}$ plane.

b) If $E_{1230}$ exists, then $E_{0230}$ is a saddle point with locally stable manifold in the $x_{2} x_{3}$ plane and with unstable manifold in the $x_{1} x_{4}$ plane.

\section{Proof}

Consider the following subsystem from (2.1)

$$
\left.\begin{array}{l}
x_{2}^{\prime}(t)=x_{2}(t)\left[b_{2}-a_{22} x_{2}(t)\right], \\
x_{3}^{\prime}(t)=x_{3}(t)\left[b_{3}-a_{33} x_{3}(t)\right] .
\end{array}\right\}
$$

Evaluating the variation matrix of system (4.4) at $E_{23}$, we have

$$
M_{23}=\left(\begin{array}{cc}
-b_{2} & 0 \\
0 & -b_{3}
\end{array}\right),
$$

which have the eigenvalues $\lambda_{1}=-b_{2}<0$ and $\lambda_{2}=-b_{3}<0$ (by using (H3)').

Therefore, $E_{23}$ is locally asymptotically stable in the $x_{2} x_{3}$ plane.

Substituting by $E_{0230}$ in the variation matrix (4.1), we get the eigenvalues

$$
\lambda_{1}=b_{1}-\frac{a_{12}}{a_{22}} b_{2}-\frac{a_{13}}{a_{33}} b_{3}, \quad \lambda_{2}=-b_{2}, \quad \lambda_{3}=-b_{3}, \quad \lambda_{4}=b_{4} .
$$

If $E_{1230}$ exists, then

$$
\lambda_{1}>0, \quad \lambda_{2}<0, \quad \lambda_{3}<0, \quad \lambda_{4}>0
$$

(By using (H3)' and (H5)').

Hence $E_{0230}$ is a saddle point with locally stable manifold in the $x_{2} x_{3}$ plane and with unstable manifold in the $x_{1} x_{4}$ plane.

\section{Theorem 4.2}

a) $E_{123}$ is locally asymptotically stable in the $x_{1} x_{2} X_{3}$ space.

b) If $E^{*}$ exists, then $E_{1230}$ is a saddle point with locally stable manifold in the $x_{1} x_{2} x_{3}$ space and with unstable manifold in the $x_{4}$ direction.

\section{Proof}

Consider the following subsystem from (2.1)

$$
\left.\begin{array}{l}
x_{1}^{\prime}(t)=x_{1}(t)\left[b_{1}-a_{11} x_{1}(t)-a_{12} x_{2}(t)-a_{13} x_{3}(t)\right], \\
x_{2}^{\prime}(t)=x_{2}(t)\left[b_{2}-a_{21} x_{1}(t)-a_{22} x_{2}(t)\right], \\
x_{3}^{\prime}(t)=x_{3}(t)\left[b_{3}-a_{31} x_{1}(t)-a_{33} x_{3}(t)\right] .
\end{array}\right\}
$$

Evaluating the variation matrix of system (4.5) at $E_{123}$, we have 


$$
M_{123}=\left(\begin{array}{ccc}
-a_{11} \hat{x}_{123} & -a_{12} \hat{x}_{123} & -a_{13} \hat{x}_{123} \\
-a_{21} \hat{x}_{23} & -a_{22} \hat{x}_{23} & 0 \\
-a_{31} \hat{x}_{32} & 0 & -a_{33} \hat{x}_{32}
\end{array}\right),
$$

which has the characteristic polynomial

$$
\lambda^{3}+c_{1} \lambda^{2}+c_{2} \lambda+c_{3}
$$

where

$$
\begin{aligned}
& c_{1}=a_{11} \hat{x}_{123}+a_{22} \hat{x}_{23}+a_{33} \hat{x}_{32}, \\
& c_{2}=a_{11} a_{22} \hat{x}_{123} \hat{x}_{23}+a_{11} a_{33} \hat{x}_{123} \hat{x}_{32}+a_{22} a_{33} \hat{x}_{23} \hat{x}_{32}-a_{12} a_{21} \hat{x}_{123} \hat{x}_{23}-a_{13} a_{31} \hat{x}_{123} \hat{x}_{32}, \\
& c_{3}=\left[a_{11} a_{22} a_{33}-a_{12} a_{21} a_{33}-a_{13} a_{31} a_{22}\right] \hat{x}_{123} \hat{x}_{23} \hat{x}_{32} .
\end{aligned}
$$

From Routh-Hurwitz criterion, $E_{123}$ is locally asymptotically stable if and only if $c_{1}>0, c_{3}>0$ and $c_{1} C_{2}>c_{3}$.

It is clear that all the coefficients $c_{1}, c_{2}$ and $c_{3}$ are positive and

$$
\begin{aligned}
c_{1} c_{2}-c_{3}= & a_{11} \hat{x}_{123}\left[a_{11} a_{22} \hat{x}_{123} \hat{x}_{23}+a_{11} a_{33} \hat{x}_{123} \hat{x}_{32}-a_{12} a_{21} \hat{x}_{123} \hat{x}_{23}-a_{13} a_{31} \hat{x}_{123} \hat{x}_{32}\right] \\
& +a_{22} \hat{x}_{23}\left[a_{11} a_{22} \hat{x}_{123} \hat{x}_{23}+a_{11} a_{33} \hat{x}_{123} \hat{x}_{32}+a_{22} a_{33} \hat{x}_{23} \hat{x}_{32}-a_{12} a_{21} \hat{x}_{123} \hat{x}_{23}\right] \\
& +a_{33} \hat{x}_{32}\left[a_{11} a_{22} \hat{x}_{123} \hat{x}_{23}+a_{11} a_{33} \hat{x}_{123} \hat{x}_{32}+a_{22} a_{33} \hat{x}_{23} \hat{x}_{32}-a_{13} a_{31} \hat{x}_{123} \hat{x}_{32}\right]>0
\end{aligned}
$$

Therefore $E_{123}$ is locally asymptotically stable in the $x_{1} x_{2} x_{3}$ space.

Substituting by $E_{1230}$ in the variation matrix (4.1), we get the characteristic equation

$$
\left[\lambda^{3}+c_{1} \lambda^{2}+c_{2} \lambda+c_{3}\right]\left(b_{4}-a_{41} \hat{x}_{123}-\lambda\right)=0 .
$$

Comparing with (4.6), we obtain that $\lambda_{1}, \lambda_{2}$ and $\lambda_{3}$ have negative real parts while $\lambda_{4}=b_{4}-a_{41} \hat{x}_{123}>0$ (by using (H6) and (H6)')

Therefore, $E_{1230}$ is a saddle point with locally stable manifold in the $x_{1} x_{2} x_{3}$ space and with unstable manifold in the $x_{4}$ direction.

Remark 4.1

1) Behavior of solutions near $E_{124}$ and $E_{134}$ are the same behavior of solutions near the equilibrium point $E_{123}$.

2) Behavior of solutions near $E_{1204}$ and $E_{1034}$ are the same behavior of solutions near $E_{1230}$.

\section{Theorem 4.3}

a) $E_{234}$ is locally asymptotically stable in the $x_{2} x_{3} x_{4}$ space.

b) If $E^{*}$ exists, then $E_{0234}$ is a saddle point with locally stable manifold in the $x_{2} X_{3} X_{4}$ space and with unstable manifold in the $x_{4}$ direction.

\section{Proof}

Proof of this theorem follows directly as proof of Theorem 4.2

Now, we study asymptotic stability of the positive equilibrium $E^{*}$.

Substituting by $E^{*}$ in the variation matrix (4.1), we get the characteristic equation

$$
\lambda^{4}+c_{1} \lambda^{3}+c_{2} \lambda^{2}+c_{3} \lambda+c_{4}=0
$$

where

$$
\begin{aligned}
c_{1}= & a_{11} x_{1}^{*}+a_{22} x_{2}^{*}+a_{33} x_{3}^{*}+a_{44} x_{4}^{*}, \\
c_{2}= & a_{11} a_{22} x_{1}^{*} x_{2}^{*}+a_{11} a_{33} x_{1}^{*} x_{3}^{*}+a_{11} a_{44} x_{1}^{*} x_{4}^{*}+a_{22} a_{33} x_{2}^{*} x_{3}^{*}+a_{22} a_{44} x_{2}^{*} x_{4}^{*} \\
& +a_{33} a_{44} x_{3}^{*} x_{4}^{*}-a_{12} a_{21} x_{1}^{*} x_{2}^{*}-a_{13} a_{31} x_{1}^{*} x_{3}^{*}-a_{14} a_{41} x_{1}^{*} x_{4}^{*}, \\
c_{3}= & a_{11} a_{22} a_{33} x_{1}^{*} x_{2}^{*} x_{3}^{*}+a_{11} a_{22} a_{44} x_{1}^{*} x_{2}^{*} x_{4}^{*}+a_{11} a_{33} a_{44} x_{1}^{*} x_{3}^{*} x_{4}^{*}+a_{22} a_{33} a_{44} x_{2}^{*} x_{3}^{*} x_{4}^{*} \\
& -a_{12} a_{21} a_{33} x_{1}^{*} x_{2}^{*} x_{3}^{*}-a_{12} a_{21} a_{44} x_{1}^{*} x_{2}^{*} x_{4}^{*}-a_{13} a_{31} a_{22} x_{1}^{*} x_{2}^{*} x_{3}^{*}-a_{13} a_{31} a_{44} x_{1}^{*} x_{3}^{*} x_{4}^{*} \\
& -a_{14} a_{41} a_{22} x_{1}^{*} x_{2}^{*} x_{4}^{*}-a_{14} a_{41} a_{33} x_{1}^{*} x_{3}^{*} x_{4}^{*}, \\
c_{4}= & \left(a_{11} a_{22} a_{33} a_{44}-a_{12} a_{21} a_{33} a_{44}-a_{13} a_{31} a_{22} a_{44}-a_{14} a_{41} a_{22} a_{33}\right) x_{1}^{*} x_{2}^{*} x_{3}^{*} x_{4}^{*} .
\end{aligned}
$$


From Routh-Hurwitz criterion [12], $E^{*}$ is locally asymptotically stable if and only if $c_{1}>0, c_{3}>0, c_{4}>0$ and $c_{1} c_{2} c_{3}>c_{3}^{2}+c_{1}^{2} c_{4}$.

It is clear that all the coefficients $c_{1}, c_{2}, c_{3}$ and $c_{3}$ are positive and if

$$
c_{1} c_{2} c_{3}-c_{3}^{2}-c_{1}^{2} c_{4}>0 .
$$

Then $E^{*}$ is locally asymptotically stable in $R_{+}^{4}$.

\section{Theorem 4.4}

$E^{*}$ is globally asymptotically stable in $R_{+}^{4}$ for every carrying capacity $b \in R^{4}$.

\section{Proof.}

We define the Liapunov function $V(x)$ by

$$
V(x)=\sum_{i=1}^{4} w_{i}\left(x_{i}-x_{i}^{*}-x_{i}^{*} \ln \frac{x_{i}}{x_{i}^{*}}\right),
$$

where $x=\left(x_{1}, x_{2}, x_{3}, x_{4}\right), \quad w_{i}>0, i=1,2,3,4$.

In the region

$$
G=\left\{\left(x_{1}, x_{2}, x_{3}, x_{4}\right) \mid x_{i}>0, i=1,2,3,4\right\} .
$$

It is clear that

$$
\begin{aligned}
& V(x) \in C^{1}(G, R), \\
& V(x)=0 \Leftrightarrow x=E^{*}, \\
& V(x)>0 \text { for } x \in G-\left\{E^{*}\right\} .
\end{aligned}
$$

Then calculating the time derivative of $V$ along the positive solutions of system (2.1), we have

$$
\begin{aligned}
\dot{V}= & -w_{1} a_{11}\left(x_{1}-x_{1}^{*}\right)^{2}-\left(w_{1} a_{12}+w_{2} a_{21}\right)\left(x_{1}-x_{1}^{*}\right)\left(x_{2}-x_{2}^{*}\right) \\
& -\left(w_{1} a_{13}+w_{3} a_{31}\right)\left(x_{1}-x_{1}^{*}\right)\left(x_{3}-x_{3}^{*}\right)-\left(w_{1} a_{14}+w_{4} a_{41}\right)\left(x_{1}-x_{1}^{*}\right)\left(x_{4}-x_{4}^{*}\right) \\
& -w_{2} a_{22}\left(x_{2}-x_{2}^{*}\right)^{2}-w_{3} a_{33}\left(x_{3}-x_{3}^{*}\right)^{2}-w_{4} a_{44}\left(x_{4}-x_{4}^{*}\right)^{2} .
\end{aligned}
$$

Then, we can choose

$$
w_{1}=1, \quad w_{j}=-\frac{a_{1 j}}{a_{j 1}}, \quad j=2,3,4
$$

Hence, we obtain

$$
\dot{V}=-a_{11}\left(x_{1}-x_{1}^{*}\right)^{2}+\frac{a_{12}}{a_{21}} a_{22}\left(x_{2}-x_{2}^{*}\right)^{2}+\frac{a_{13}}{a_{31}} a_{33}\left(x_{3}-x_{3}^{*}\right)^{2}+\frac{a_{14}}{a_{41}} a_{44}\left(x_{4}-x_{4}^{*}\right)^{2}<0 .
$$

Therefore, it follows from well-known Liapunov-LaSalle theorem that the positive equilibrium $E^{*}$ is globally asymptotically stable in $R_{+}^{4}$.

\section{Numerical Simulations}

The reader can be check local asymptotic stability of the system 2.1 for:

\section{Example 5.1}

$$
\begin{aligned}
& b_{1}=a_{12}=a_{22}=1, \quad a_{11}=a_{13}=a_{44}=0.5, \quad a_{33}=a_{14}=0.25 \\
& a_{21}=-1, \quad a_{31}=b_{2}=-0.75, \quad a_{41}=b_{3}=-0.5
\end{aligned}
$$

\section{Example 5.2}

$$
\begin{aligned}
& b_{1}=-1, \quad a_{11}=1, \quad a_{22}=a_{41}=a_{44}=b_{3}=0.5, \quad a_{21}=a_{33}=b_{4}=0.25, \\
& a_{31}=b_{2}=0.75, \quad a_{12}=-0.75, \quad a_{13}=-0.5, \quad a_{14}=-0.25
\end{aligned}
$$




\section{Acknowledgements}

The authors would like to thank all staff members who help me in this article.

\section{References}

[1] May, R.M. (1973) Stability and Complexity in Model Ecosystems. Princeton University Press, Princeton.

[2] Edelstein-Keshet, L. (2005) Mathematical Models in Biology. Society for Industrial and Applied Mathematics, New York. http://dx.doi.org/10.1137/1.9780898719147

[3] Farkas, M. Dynamical Models in Biology. Elsevier Science and Technology Books, 200.

[4] Freedman, H.I. (1980) Deterministic Mathematical Models in Population Ecology. Marcel Dekker, Inc., New York.

[5] Murray, J.D. (2002) Mathematical Biology, Interdisciplinary Applied Mathematics. Springer, Berlin.

[6] Perthame, B. (2007) Transport Equations in Biology. Birkhuuser Verlag, Basel.

[7] Solimano, F. and Berettra, E. (1982) Graph Theoretical Criteria for Stability and Boundedness of Predator-Prey System. Bulletin of Mathematical Biology, 44, 579-585. http://dx.doi.org/10.1137/1.9780898719147

[8] Takeuchi, Y., Adachi, N. and Tokumaru, H. (1978) The Stability of Generalized Volterra Equations. Journal of Mathematical Analysis and Applications, 62, 453-473. http://dx.doi.org/10.1016/0022-247X(78)90139-7

[9] Ji, X.-H. (1996) The Existence of Globally Stable Equilibria of N-Dimensional Lotka-Volterra Systems. Applicable Analysis: An International Journal, 62, 11-28. http://dx.doi.org/10.1080/00036819608840467

[10] Arrowsmith, D.K. and Place, C.M. (1982) Ordinary Differential Equation. Chapman and Hall, New York.

[11] Li, X.-H., Tang, C.-L and Ji, X.-H. (1999) The Criteria for Globally Stable Equilibrium in N-Dimensional Lotka-Volterra Systems. Journal of Mathematical Analysis and Applications, 240, 600-606. http://dx.doi.org/10.1006/jmaa.1999.6612

[12] Lu, Z. (1998) Global Stability for a Lotka-Volterra System with a Weakly Diagonally Dominant Matrix. Applied Mathematics Letters, 11, 81-84. http://dx.doi.org/10.1016/S0893-9659(98)00015-9

[13] Liu, J. (2003) A First Course in the Qualitative Theory of Differential Equations. Person Education, Inc., New York.

[14] Takeuchi, Y. and Adachi, N. (1980) The Existence of Globally Stable Equilibria of Ecosystems of the Generalized Volterratyp. Journal of Mathematical Biology, 10, 401-415. http://dx.doi.org/10.1007/BF00276098

[15] Takeuchi, Y. Adachi, N. (1984) Influence of Predation on Species Coexistence in Volterra Models. Journal of Mathematical Biology, 70, 65-90. http://dx.doi.org/10.1016/0025-5564(84)90047-6

[16] Takeuchi, Y. (1996) Global Dynamical Properties of Lotka-Volterra Systems. World Scientific, Singapore City. http://dx.doi.org/10.1142/9789812830548

[17] Rao, M. (1980) Ordinary Differential Equations Theory and Applications. Pitman Press, Bath. 